\title{
The identification of a spontaneous 47, XX, $+21 / 46$, XY chimeric fetus with male genitalia
}

\author{
Kuei-Fang Lee ${ }^{1,2+}{ }^{+}$Chun-Shuo Hsu ${ }^{3+}$, Pao-Lin Kuo ${ }^{4}$, Jing-Liang Chen ${ }^{5}$, Yuan-Hong Jiang ${ }^{5}$ and Ingrid Y Liu ${ }^{6 *}$
}

\begin{abstract}
Background: Approximately 30 sex-chromosome discordant chimera cases have been reported to date, of which only four cases carried trisomy 21 . Here, we present an additional case, an aborted fetus with a karyotype of $47, X X,+21 / 46, X Y$.

Case presentation: Autopsy demonstrated that this fetus was normally developed and had male genitalia. Major characteristics of Down syndrome were not observed except an enlarged gap between the first and second toes. Karyotyping of tissues cultured from the fetus revealed the same chimeric chromosomal composition detected in the amniotic fluid but with a different ratio of $[47, X X,+21]$ to $[46, X Y]$. Further short tandem repeat analysis indicated a double paternal contribution and single maternal contribution to the fetus, with the additional chromosome 21 in the $[47, X X,+21]$ cell lineage originating from the paternal side.
\end{abstract}

Conclusion: We thus propose that this chimeric fetus was formed via the dispermic fertilization of a parthenogenetic ovum with one $(Y)$ sperm and one $(X,+21)$ sperm.

Keywords: Chimerism, Trisomy 21, Sex chromosome, Fetus, Genitalia, Karyotype

\section{Background}

Sex-chromosome discordant chimeras $(46, \mathrm{XX} / 46, \mathrm{XY})$ are extremely rare in humans; in fact, its incidence is undetermined [1]. Gartler et al. described the first case of sex-chromosome discordant chimera as well as a hermaphrodite (with ambiguous genitalia) formed via double fertilization in 1962 [2], and approximately 30 cases have been reported since [3]. There are especially few reported cases of chimerism involving coexisting normal and abnormal lineages that each carries a distinct sex chromosome complement. Only four cases of sex-chromosome discordant chimerism with trisomy 21 have been reported to date. One was a true hermaphrodite [4], one was a newborn infant with ambiguous genitalia [5], and other two cases had normal gonads [6,7]. Three cases harbored an extra chromosome 21 in their XY lineages [4-6], and one case had mosaicism in the XX lineage [7]. Here, we present the fifth case of sex-chromosome

\footnotetext{
* Correspondence: ycliu@mail.tcu.edu.tw

${ }^{\dagger}$ Equal contributors

${ }^{6}$ Department of Molecular Biology and Human Genetics, Tzu Chi University, 701, Sec 3, Chunyang Rd, Hualien 970, Taiwan

Full list of author information is available at the end of the article
}

discord4nt chimerism, with trisomy $21(47, \mathrm{XX}+21 / 46$, $\mathrm{XY}$ ) and male genitalia.

\section{Case presentation}

A phenotypically normal 21-year-old female with an obstetric history of gravida 1 and para 0 (G1P0) requested an amniocentesis at the $18^{\text {th }}$ week of gestation because of anxiety. Although prenatal ultrasound examinations appeared normal, amniocentesis revealed a sex chromosome-discordant chimeric karyotype with trisomy 21 in the $\mathrm{XX}$ lineage $(47, \mathrm{XX},+21 / 46, \mathrm{XY})$. After receiving genetic counselling, she and her non-consanguineous husband, a 29-year-old with acquired mutism caused by suspected viral infection at the age of 7 months, decided to terminate the pregnancy via induced vaginal delivery at the $21^{\text {st }}$ week. They agreed to donate their blood and the aborted fetus for further medical research.

\section{Methods \\ Autopsy}

The aborted fetus was $15.5 \mathrm{~cm}$ in body length and weighed $262 \mathrm{~g}$. The head, trunk, and extremities appeared grossly normal. Autopsy was performed according to standard procedures. Tissues from brain, kidneys, skin,

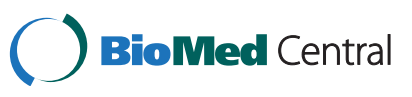


placenta, and cord blood were collected for karyotyping. DNA wa4 isolated directly from the brain for further genetic analysis.

\section{Karyotypig and preparation of genomic DNA}

Karyotying was performed as standard protocol. Genomic DNA was purified according to the manual of DNA isolation kit produced by Gentra system,USA.

\section{Short-tandem-repeat genotyping}

Short-tandem-repeat genotyping was performed according to the manual of Human Sex and Autosomal STR Mapping Set (GenePhile G-Plex Kit, GenePhile Bioscience Co., Ltd, Taiwan). A total of 16 STR markers were applied: AMEL amplifies different length of fragments from $X$ and $\mathrm{Y}$ chromosome and the remaining 15 are autosomal markers, of which one is on chromosome 21 (D21S1437). Additional 3 STR markers specific to chromosome 21 were obtained from AmpFISTR ${ }^{\circledR}$ Identifiler ${ }^{\mathrm{Tm}}$ PCR Amplification Kit (Applied Biosystems, Foster City, CA, USA) (D21S11) and Bioscience Co., Ltd (D21S1436 and D21S1270). GenePhile G-Plex PCR Amplification Kit (GenePhile Bioscience Co., Ltd, Taiwan) was used for STR genotyping. Genotypes were scored using Gene Scan ${ }^{\circledR}$ and Genotyper ${ }^{\circledR}$ softwares (Applied Biosystems, USA) and were verified blindly by three technicians.

\section{Ethics statement}

This research was performed abiding by the regulation of the institutional review board (IRB) case 098-82 of the Tzu-Chi general hospital. Written informed consent has been obtained from the parents for publication of this case report and accompanying images.

\section{Results}

\section{Morphological examination of the fetus}

The morphologies of the fetal head, face, trunk, and extremities appeared to be grossly normal, as did the internal organs, including the heart, lungs, gastrointestinal, hepatobiliary, and genitourinary systems. External genitalia and gonads revealed normal male development, though bilateral cryptorchidism was observed at this embryonic stage (Figure 1A). Characteristics of Down syndrome were not obvious except for an enlarged gap between the first and the second toes (Figure 1B).

\section{Karyotypes}

Chromosomal analyses of the parents revealed normal karyotypes (figures not shown). Karyotypes obtained from various cultured fetal tissues indicated the presence of sex-chromosome discordant trisomy 21 chimerism but with different ratios of $[47, \mathrm{XX},+21]$ to $[46, \mathrm{XY}]$. Twenty cells from the cortex and placenta and sixteen
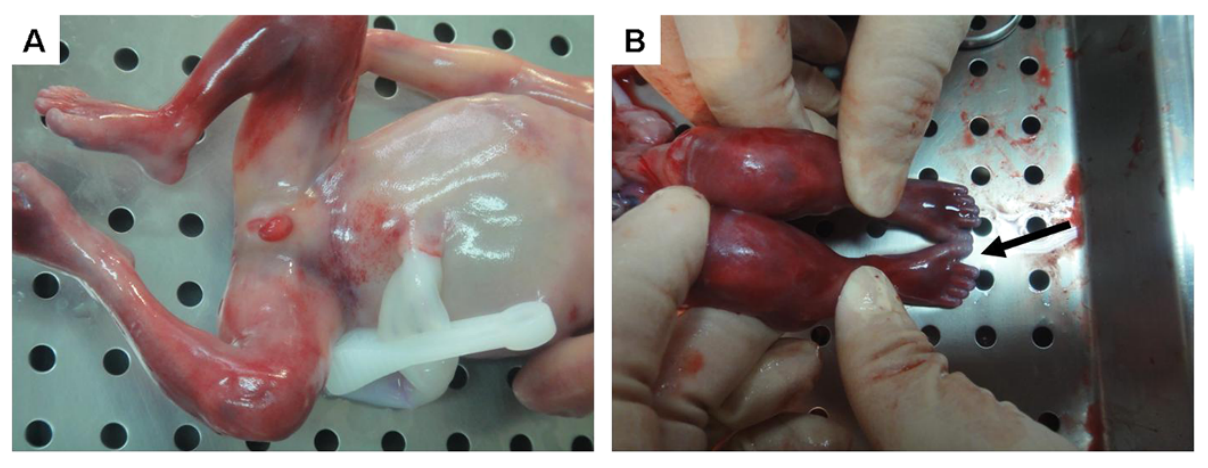

C

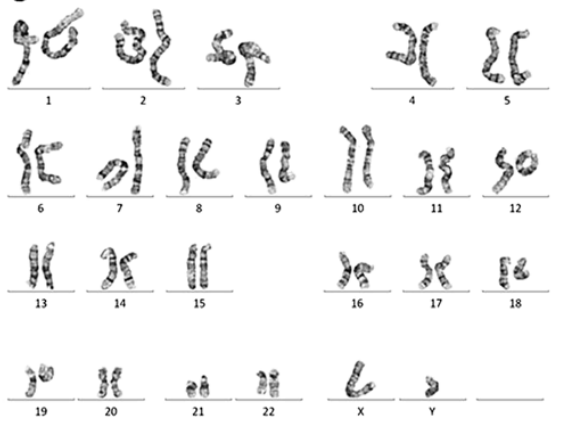

D
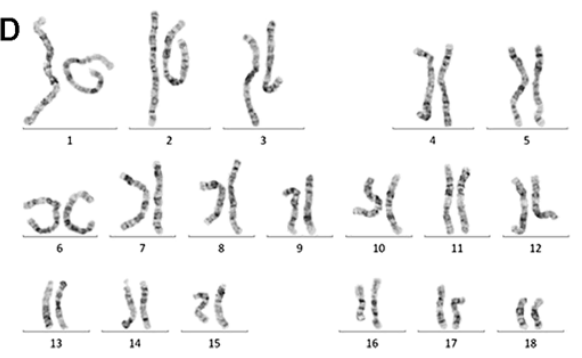

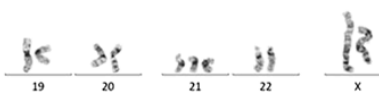

Figure 1 Autopsy and karyotypes of the chimera fetus. The aborted fetus exhibits normal external genitalia (A) and no characteristics typical of Down syndrome except the enlarged gap between the first and the second toes (B) was observed. The fetal karyotypic ratio of 46,XY (C) to 47, $\mathrm{XX},+21(\mathbf{D})$ in cortical cells was 11:9. 
Table 1 Chromosome map position for STR markers used in the present report

\begin{tabular}{|c|c|c|c|c|c|c|c|c|}
\hline \multirow{3}{*}{$\begin{array}{l}\text { STR marker } \\
\text { D2S1338 }\end{array}$} & \multirow{3}{*}{$\begin{array}{l}\text { Chr. Position } \\
2\end{array}$} & \multirow{2}{*}{\multicolumn{2}{|c|}{$\begin{array}{c}\text { Father } \\
\text { map position }\end{array}$}} & \multirow{2}{*}{\multicolumn{2}{|c|}{$\begin{array}{c}\text { Mother } \\
\text { map position }\end{array}$}} & \multirow{2}{*}{\multicolumn{3}{|c|}{$\begin{array}{c}\text { Fetus } \\
\text { map position }\end{array}$}} \\
\hline & & & & & & & & \\
\hline & & 319.28 & 327.46 & 315.19 & 347.38 & 319.54 & 347.51 & 327.90 \\
\hline TPOX & 2 & 229.54 & 241.52 & 241.55 & & 229.67 & 241.48 & \\
\hline D3S1358 & 3 & 128.06 & & 123.98 & 127.99 & 128.15 & 124.02 & \\
\hline FGA & 4 & 226.14 & 234.13 & 238.14 & 246.29 & 234.24 & 238.31 & \\
\hline D5S818 & 5 & 155.13 & & 146.81 & 159.47 & 155.26 & 159.47 & \\
\hline CSF1PO & 5 & 316.83 & 325.03 & 316.93 & 321.10 & 317.07 & 321.28 & 325.46 \\
\hline D7S820 & 7 & 278.62 & & 270.62 & 278.77 & 270.71 & 278.77 & \\
\hline D8S1179 & 8 & 148.08 & 160.89 & 135.51 & 139.75 & 148.22 & 135.54 & 161.01 \\
\hline Tho1 & 11 & 170.87 & 182.80 & 182.87 & & 170.87 & & 182.80 \\
\hline vWA & 12 & 178.41 & 186.30 & 178.50 & & 178.54 & 186.43 & \\
\hline D13S317 & 13 & 216.40 & 220.42 & 224.49 & 232.40 & 216.58 & 232.40 & \\
\hline D16S539 & 16 & 271.91 & 275.88 & 275.91 & 283.97 & 271.93 & 276.03 & \\
\hline D18S51 & 18 & 294.27 & & 290.16 & 307.19 & 294.41 & 307.26 & \\
\hline D19S433 & 19 & 121.71 & & 121.68 & & 121.72 & & \\
\hline D21S11 & 21 & 203.82 & 207.78 & 217.67 & & 203.93 & 217.74 & \\
\hline D21S1270 & 21 & 242.42 & 268.84 & 258.65 & 266.83 & 242.38 & 258.69 & 268.84 \\
\hline D21S1437 & 21 & 111.45 & 123.48 & 127.49 & & 111.42 & 127.47 & 123.43 \\
\hline D21S1436 & 21 & 174.55 & 190.29 & 174.54 & & 174.49 & 190.29 & \\
\hline \multirow[t]{2}{*}{ AMEL } & $x$ & & 106.43 & & 106.38 & & 106.51 & \\
\hline & Y & & 112.22 & & & & 112.28 & \\
\hline
\end{tabular}

cells from the skin and kidney were analyzed. The ratio of $[46, \mathrm{XY}]$ (Figure 1C) to $[47, \mathrm{XX},+21]$ (Figure 1D) was 11:9 in the cortex and placenta and 11:5 in the skin and kidney.

\section{STR analysis}

To clarify mechanism of the chimera formation and identify the origin of the additional chromosome 21, we used 19 markers (Table 1) to perform STR analyses on samples from the parents and fetus. Only 6 of the 19 markers were informative (including AMEL); 4 of the informative markers localized to chromosomes $\mathrm{Y}, 2$ (D2S1338), 5 (CSF1PO), 8 (D8S1179), and 2 localized to chromosome 21 (DS21S1270 and D21S1437). The sex chromosome-specific marker amelogenin (AMEL) indicated disomic sex chromosomes (Figure 2A). The autosomal markers D2S1338, CSF1PO, D8S1179 (Figure 2A) and chromosome 21 markers D21S1270, D21S1437 (Figure 2B) indicated that the fetus inherited two (heterozygous) alleles from the father and one allele from the mother. STR analyses identified a double paternal contribution (including chromosome 21) and one maternal contribution to the fetal genotype.

\section{Conclusion}

Several mechanisms may be involved in the formation of an XX/XY chimera: 1) a tetragametic chimera results from the post-zygotic fusion of two distinct embryos; 2) a chimera results from the dispermic fertilization of an self -replicated oocyte or the second polar body; 3) an androgenetic chimera (isodisomic paternal cell lines) occurs when one normal zygote fuses with another zygote formed via one sperm fertilizing with an egg that is empty of genetic material (for a review, please see [1]). In addition, a post-zygotic diploidization and nondisjunction of chromosome 21 of a triploid is also possible. In cases of tetragametic chimeras, both double paternal and double maternal chromosome contributions would be detected. Recently, in vitro fertilization appears to increase the incidence of tetragametic chimeras $[8,9]$ because standard practices necessitate the utilization of more than one embryo to increase success rates. Molecular analyses have been performed for 2 [4,7] of the 4 previously reported cases with sex chromosome discordant chimerism with trisomy 21 . Both were tetragametic chimeras and revealed a paternal origin of the extra $21^{\text {st }}$ chromosome. Sex-chromosome discordant 
A
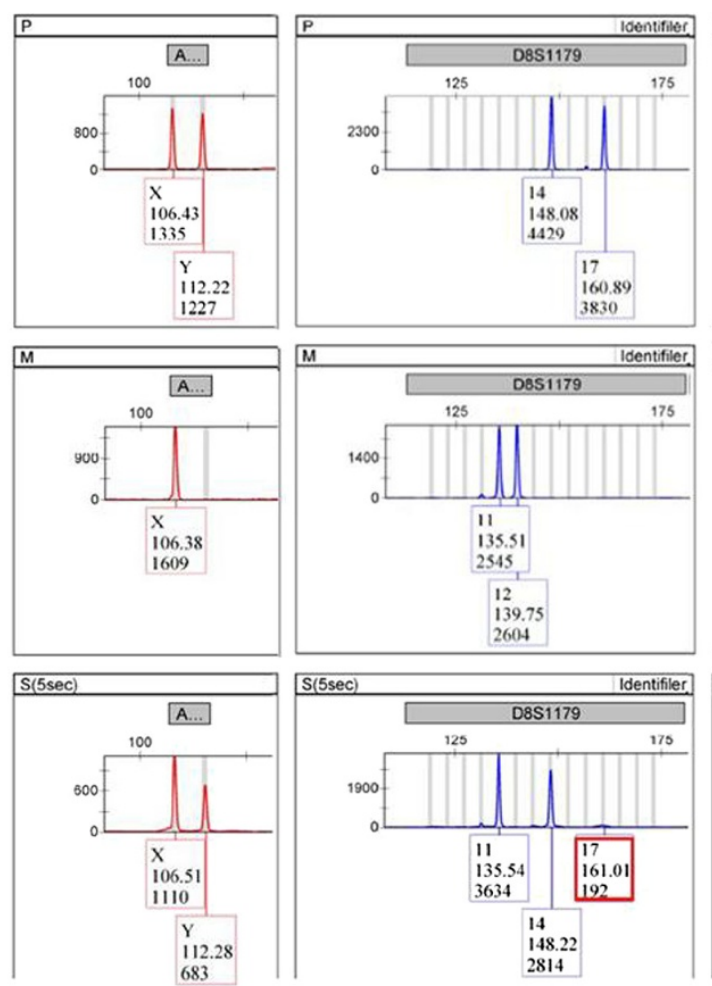
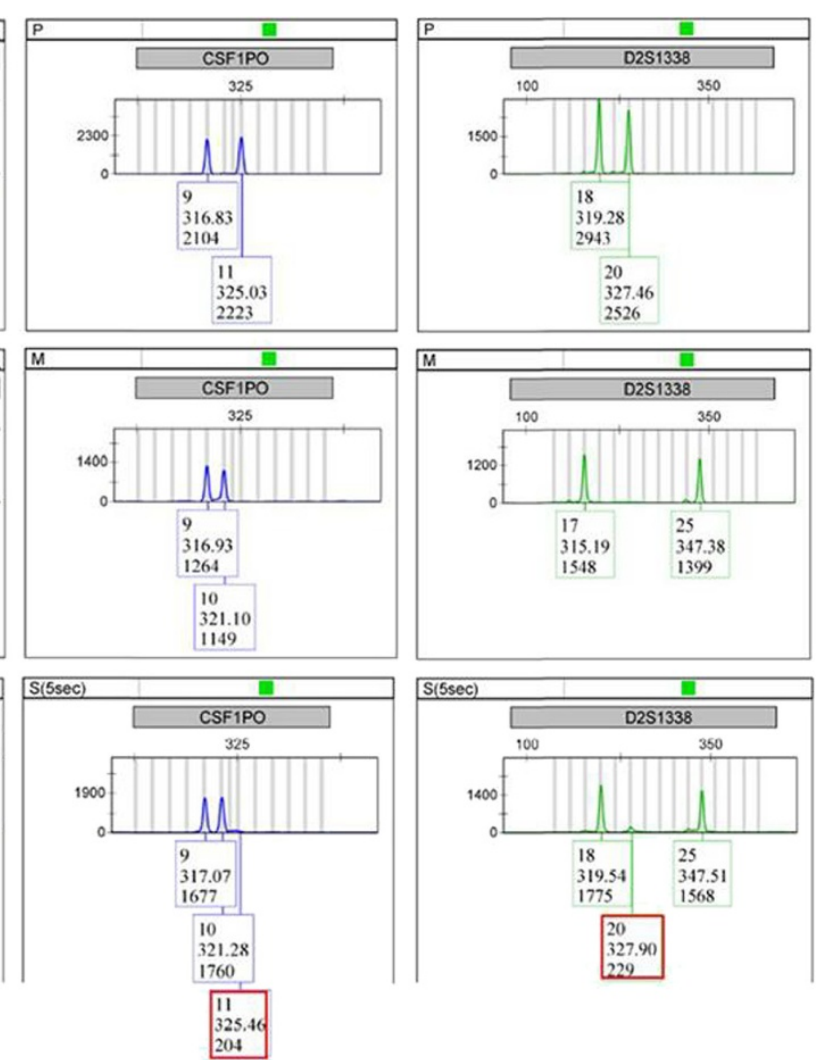

B
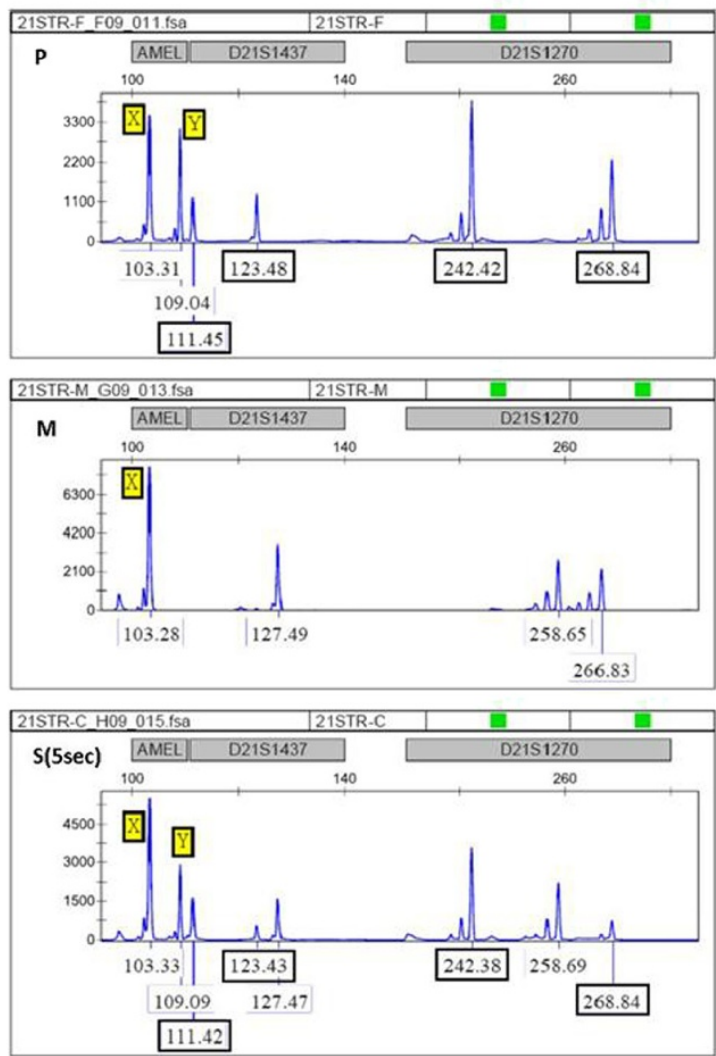

Figure 2 (See legend on next page.) 
tetragametic chimera can also be fused from two abnormal cell linages. A stillborn male fetus with multiple congenital anomalies was identified to have a $(47, \mathrm{XY},+21$ / $47, \mathrm{XX},+12)$ karyotype [10]. In chimera cases that result from double fertilization of a self-replicated egg or the second polar body, a double paternal contribution and single maternal contribution would be detected. According to our STR analysis, this is the putative mechanism that led to the genotype of the present case. This fetus may have been formed from a parthenogenicallyactivated oocyte fertilized with two sperms with opposite sex chromosomes, one of which harbored an extra chromosome 21 (Figure 3). The possibility of double fertilization with the second polar body is considered very rare because there is a lack of evidence of crossing over events, which requires further analysis with more STR markers. In addition, the coexistence of both $(47, \mathrm{XX},+21)$ cells and $(46, \mathrm{XY})$ cells in the present case were detected in various tissues originating from different germ layers, indicating that the formation of this chimeric fetus occurred at an extremely early embryonic development stage. A sex-chromosome discordant chimerism with ambiguous genitalia and trisomy 14 in the $\mathrm{XY}$ cell linage $(46, \mathrm{XX} / 47, \mathrm{XY},+14)$ was reported to form via similar mechanism [11].

The prenatal detection rate of $46, \mathrm{XX} / 46, \mathrm{XY}$ from heterogeneous amniotic cells is approximately $0.24 \%$ [12]. Maternal cell contamination of the male fetal amniotic fluid accounts for the large majority of cases. Other cases of 46, XX/46, XY may result from laboratory error due to cross-contamination between two samples [3]. The death of a twin would not result in coexistence of $\mathrm{XX}$ and XY cells in the amniotic fluid because cells in dead tissues stop dividing and would not be detected by karyotyping [12]. Thus, it is only after excluding these possibilities that the prenatal diagnosis of chimerism or mosaicism may be considered [1]. Most sexchromosome discordant chimeras $(\mathrm{XX} / \mathrm{XY})$ are diagnosed due to the presence of ambiguous genitalia at birth, and these individuals account for approximately $13 \%$ of true hermaphrodites. Phenotypic expression is highly variable for sex-chromosome discordant chimeras and may range from normal to ambiguous external genitalia. Those with ambiguous genitalia (hermaphrodites) may develop normally and even reproduce successfully [1]. Most sex-chromosome discordant chimerism cases demonstrate normal cognitive function. However, sexual ambiguity, infertility and the possibility of developing gonadoblastomas are major concerns in these individuals. At an early embryonic stage, chimeras with trisomy 21 appear to be grossly normal, as was observed in our case and the case that was reported by Hwa et al. [7]. However, it is difficult to predict whether such cases would develop normally at later stages. The phenotypic expressions of such cases are variable. One case was shown to exhibit multiple congenital defects and anencephaly [6], another presented with ambiguous genitalia [5], and one exhibited with ambiguous genitalia but no clinical features of Down syndrome [4]. Genetic counseling for sex-chromosome discordant chimeras with or without trisomy 21 can be extremely difficult. Informative counseling regarding cognitive function, sexual ambiguity, infertility, and the possibility of developing gonadoblastomas should be provided.

\section{Consent}

This research was performed abiding by the regulation of the institutional review board (IRB) case 098-82 of

Chimera resulting from double fertilization of the parthenogenetic egg

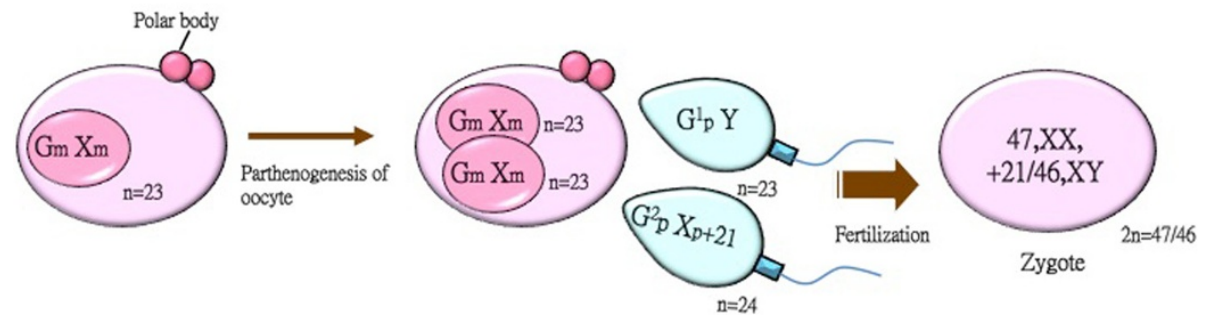

Figure 3 The mechanism of the formation of the present chimeric case with trisomy 21. This chimeric case could result from the fertilization of a parthenogenetic egg with two sperms, one $(Y)$ sperm and one $(X,+21)$ sperm. 
the Tzu-Chi general hospital. Written informed consent has been obtained from the parents for publication of this case report and accompanying images.

\section{Competing interests}

The authors declare that they have no competing interests.

\section{Authors' contribution}

K.-F. L carried out cytogenetic and molecular cytogenetic experiments. C.-S. H. carried out pedigree analysis, clinical examination and diagnosis of this family. J.-L. C. and Y.-H. J. performed anatomical examination and drafted "Patient and clinical examination" section. P.-L. K. performed STR analyses. I.Y. $L$. conceived of the study, and participated in its design, coordination and manuscript writing. All authors read and approved the final manuscript.

\section{Acknowledgements}

This study was supported by Ministry of Economic Affairs, Taiwan (grant 98EC-17-A-19-S2-0110 to I.-Y. Liu). We are grateful to Dr. J.-S. Feng, Dr. H.-C. Kuo and P.-Y. Woon for helpful comments on the manuscript. We also appreciate Miss C-W Chang, Tzu-Ying Li, Chung-Nan Yeh, Yi-Chieh Su, W-H Wang, and $\mathrm{P}-\mathrm{H}$ Hsu for technical support and manuscript preparation.

\section{Author details}

${ }^{1}$ Laboratory for Cytogenetics, Center for Genetic Counseling, Buddhist Tzu Chi General Hospital, 707, Sec 3, Chunyang Rd, Hualien 970, Taiwan.

${ }^{2}$ Graduate Institute of Medical Sciences, Tzu Chi University, Hualien County 970, Taiwan. ${ }^{3}$ Department of Obstetrics \& Gynecology, Buddhist Tzu Chi General Hospital, Dalin Branch, Chiayi County 622, Taiwan. ${ }^{4}$ Department of Medical Laboratory Science and Biotechnology, National Cheng Kung University, 1, University Road, Tainan City 701, Taiwan. ${ }^{5}$ Department of Urology, Buddhist Tzu Chi General Hospital, 707, Sec 3, Chunyang Rd, Hualien County 970, Taiwan. ${ }^{6}$ Department of Molecular Biology and Human Genetics, Tzu Chi University, 701, Sec 3, Chunyang Rd, Hualien 970, Taiwan.

Received: 12 April 2012 Accepted: 13 September 2012

Published: 20 September 2012

\section{References}

1. Malan V, Vekemans M, Turleau C: Chimera and other fertilization errors. Clin Genet 2006, 70(5):363-373.

2. Gartler SM, Waxman SH, Giblett E: An XX/XY human hermaphrodite resulting from double fertilization. Proc Natl Acad Sci U S A 1962, 48:332-335.

3. Malan V, Gesny R, Morichon-Delvallez N, Aubry MC, Benachi A, Sanlaville D, Turleau C, Bonnefont JP, Fekete-Nihoul C, Vekemans M: Prenatal diagnosis and normal outcome of a $46, X X / 46, X Y$ chimera: a case report. Hum Reprod 2007, 22(4):1037-1041.

4. Ramsay M, Pfaffenzeller W, Kotze E, Bhengu L, Essop F, de Ravel T: Chimerism in black southern African patients with true hermaphroditism 46, XX/47XY,+21 and 46, XX/46, XY. Ann N Y Acad Sci 2009, 1151:68-76.

5. Sawai T, Yoshimoto M, Kinoshita E, Baba T, Matsumoto T, Tsuji $Y$, Fukuda $S$, Harada N, Niikawa N: Case of 46, XX/47, XY, +21 chimerism in a newborn infant with ambiguous genitalia. American journal of medical genetics 1994, 49(4):428-430.

6. Danielle $R$, Lucon LMZaDPC: Chimerism $47, X Y,+21 / 46, X X$ in a female infant with anencephaly and other congenital defects. Genetics and Molecular Biology 2006, 29(1):36-37.

7. Hwa HL, Ko TM, Huang $\mathrm{CH}_{\text {, }}$ Chang LS: Prenatal diagnosis and genetic analysis of a fetus with $47, X X,+21 / 46, X X$ mosaicism and $X X / X Y$ chimerism. J Formos Med Assoc 2006, 105(8):659-663.

8. Bonthron D: Amalgamation of in vitro fertilized embryos resulting in birth of a true hermaphrodite chimera. Am J Hum Genet 1997, 61:A147.

9. Strain L, Dean JC, Hamilton MP, Bonthron DT: A true hermaphrodite chimera resulting from embryo amalgamation after in vitro fertilization. N Engl J Med 1998, 338(3):166-169.

10. Wiley JE, Madigan M, Christie JD, Smith AW: Dispermic chimerism with two abnormal cell lines, $47, X Y,+21$ and $47, X X,+12$. American journal of medical genetics 2002, 107(1):64-66.
11. Winberg J, Gustavsson P, Lagerstedt-Robinson K, Blennow E, Lundin J, Iwarsson E, Nordenstrom A, Anderlid BM, Bondeson ML, Nordenskjold A, et al: Chimerism resulting from parthenogenetic activation and dispermic fertilization. American journal of medical genetics Part A 2010, 152A(9):2277-2286.

12. Hsu LY: Prenatal diagnosis of chromosomal abnormalities through amniocentesis. In Genetic Disorders and the Fetus: Diagnosis, Prevention and Treatment. 179th edition. Baltimore, MD: Johns Hopkins University; 1998.

doi:10.1186/1471-2350-13-85

Cite this article as: Lee et al:: The identification of a spontaneous $47, \mathrm{XX}$, $+21 / 46$, XY chimeric fetus with male genitalia. BMC Medical Genetics 2012 13:85.

\section{Submit your next manuscript to BioMed Central and take full advantage of:}

- Convenient online submission

- Thorough peer review

- No space constraints or color figure charges

- Immediate publication on acceptance

- Inclusion in PubMed, CAS, Scopus and Google Scholar

- Research which is freely available for redistribution

Submit your manuscript at www.biomedcentral.com/submit
() Biomed Central 